\title{
The Worth of a Quality-Adjusted Life-Year in Patients with Diabetes: An Investigation Study using a Willingness-to-Pay Method
}

\author{
Najme Moradi ${ }^{1}$ - Arash Rashidian ${ }^{2} \cdot$ Shirin Nosratnejad ${ }^{3} \cdot$ Alireza Olyaeemanesh $^{4,5} \cdot$ Marzieh Zanganeh $^{6}$. \\ Leila Zarei ${ }^{7}$
}

Published online: 8 January 2019

(c) The Author(s) 2019

\begin{abstract}
Background A limited number of studies have specifically examined the value of quality-adjusted life-years (QALYs) from the patient's perspective.

Objective The goal of this study was to investigate the worth of QALYs from the perspectives of patients with diabetes using health and willingness-to-pay (WTP) measures.

Methods A hypothetical treatment characterized by a permanent cure was presented to 149 patients with diabetes in Tehran, Iran, to elicit the monetary value that they attach to QALYs. The QALY gains of the participants were determined using the EuroQol-5 Dimensions, 3 Levels instrument, the visual analogue scale, and the time trade-off method. A mixed closed-ended WTP model supported by an open-ended question was used to ascertain the monetary value of a QALY gained. Finally, we used each respondent's ratio of WTP to QALY gained and the mean of the ratios to estimate the worth of a QALY to all respondents.

Results In total, $96 \%$ of respondents were willing to pay out of pocket for the restoration of full health, whereas $4 \%$ exhibited a zero WTP because of an inability to pay. The mean WTP per QALY varied depending on the health measure and discount rate used, ranging from \$US1191 to \$US5043 in sensitivity analysis, which is equal to 0.23-0.95 of Iran's gross domestic product (GDP) per capita in 2015.

Conclusion Applying the upper limit of the World Health Organization's (WHO) cost-effectiveness threshold (i.e., three times the local GDP per capita) in resource allocation decisions requires caution and investigation, particularly in low- and middle-income countries with limited healthcare resources. To generalize our findings, especially for application to decision making, additional surveys involving more representative samples from different settings are recommended.
\end{abstract}

Electronic supplementary material The online version of this article (https://doi.org/10.1007/s41669-018-0111-2) contains supplementary material, which is available to authorized users.

Najme Moradi

najme.moradi@gmail.com

Arash Rashidian

arash.rashidian@gmail.com

Shirin Nosratnejad

sh_nosratnejad@yahoo.com

Alireza Olyaeemanesh

arolyaee@gmail.com

Marzieh Zanganeh

m.zan1976@gmail.com

Leila Zarei

leilazarei89@gmail.com

Extended author information available on the last page of the article

\section{Key Points for Decision Makers}

Decision makers can use the monetary value of qualityadjusted life-years (QALYs) as a threshold value for the allocation of scarce resources in reimbursement decisions.

This investigation showed that patients with diabetes were willing to pay out of pocket for the restoration of full health. Their maximum value was close to one times the local gross domestic product (GDP) per capita.

Employing the upper bound recommended by the World Health Organization (i.e., less than three times the GDP per capita) requires caution and further investigation. 


\section{Introduction}

Motivated by the aim of maximizing health and efficiency, health policy planners and decision makers around the world have shown increasing interest in using economic evaluation analyses, particularly cost-effectiveness analysis [1-3]. Recent years have also seen a rapid increase in the number of studies estimating the value of qualityadjusted life-years (QALYs) as a threshold for assessing analysis results and implementing reimbursement decisions [4-6]. Most estimation studies are typically conducted through surveys and the elicitation of QALY values from the perspectives of the general public [7, 8], but a small proportion are also based on the perspectives of patients; in some cases, a combination of these two approaches are used [9]. Studies have uncovered differences in perspectives, which may be traced to the argument that drivers of health state valuation can directly affect estimations of utility values and potentially influence resource allocation decisions [10]. Whether patients attach lower values to their health states or overestimate their willingness to obtain health benefits compared with the general public with no vested interest is under debate $[11,12]$. However, King et al. [13] showed that the monetary value of QALYs empirically derived from diverse patient groups through different preference-based methods were mostly lower than the proposed threshold values currently used as references for reimbursement decisions.

Considering the limited number of patient-based estimations [9, 13-18], we believe a need exists for more patient-oriented studies to assist informed decision making, particularly those involving estimations related to diseases that impose considerable burdens on individuals and health systems globally. Diabetes is one such disease, as its high incidence and prevalence, associated life-threatening and disabling complications, and high costs for control and treatment have rendered it "a common, growing, serious, and costly public health problem" [19]. Diabetes has directed the attention of health policy makers toward carrying out more research on the economics of diabetes management with the efficient use of limited healthcare resources [20]. To the best of our knowledge, most diabetes studies to date have focused on the clinical aspects, costs of illness, health-related quality of life, or economic evaluations, and none have specifically examined the value of QALYs from the perspectives of patients with diabetes. In consideration of this gap in the literature, we investigated the worth of QALYs among people with diabetes in Iran to determine the value of QALYs gained from diabetic treatments on the basis of stated willingness to pay (WTP) and to explore the effects of patients' characteristics on their WTP for QALYs [21, 22].

\section{Methods}

\subsection{Design}

This cross-sectional exploratory study involved face-toface interviews with patients with diabetes to estimate their WTP for QALYs. Widely used instruments for determining health preferences were employed to measure health utility weights, and a WTP model was then used to elicit the value that the patients attached to a QALY.

\subsection{Sample}

The sample comprised patients with diabetes who sought treatment in the diabetes clinic of Taleghani Hospital between June and August 2015. Taleghani Hospital is a large publicly funded educational hospital affiliated with Shahid Beheshti University of Medical Sciences, which provides healthcare services in a range of specializations, including diabetes. The inclusion criteria were patients aged $\geq 18$ years with type 1 diabetes mellitus (T1DM) or type 2 diabetes mellitus (T2DM) and able to understand and speak Farsi. We excluded women with gestational diabetes mellitus and patients with serious health problems, such as foot amputation, blindness, or severe kidney failure; the latter were excluded given the nature of the time trade-off (TTO) questions. The study protocol was approved by the university ethics committee, and all patients completed written informed consent forms before interviews were conducted.

\subsection{Data Collection}

The face-to-face interviews were carried out by a trained expert using a structured questionnaire, which is available in the Electronic Supplementary Material (ESM).

\subsubsection{Questionnaire Development}

The questionnaire consists of an introduction then sections with questions regarding health-utility measures, WTP, patient characteristics, and disease-related variables.

2.3.1.1 Utility Measures Calculating QALYs requires two sets of data: health-utility weights associated with a patient's health state (scaled from 0 to 1 ), and the length of time spent in a given target health state. This study measured patients' health-utility weights using the EuroQol-5 Dimensions, 3 Levels (EQ-5D-3L) instrument, the visual analogue scale (VAS), and the TTO method. TTO is the most widely used technique for eliciting current health-state preferences and involves presenting individuals with a scenario wherein they are asked to choose between lifespan and quality of life. For 
example, respondents are asked how much time they are willing to sacrifice for a shorter lifespan in full health. The utility of health is equal to the ratio between the duration of full health and the duration of a current health state [23]. In this study, patients were presented with two TTO scenarios according to timeframe. The first featured minimally adjusted expected lifetimes for the patients' age groups; the adjustments were made on the basis of Iran's life table [24]. For example, the expected remaining lifetime would be 50 years for a respondent aged 20-29 and 40 years for one aged 30-39 years. The second scenario was a conventional TTO scenario wherein the patients were presented with a timeframe of 10 years for trade-off. In both scenarios, patients were asked which situation they would prefer: to live out the remainder of their lives (10 years) under their current health state or to live a shorter duration but at full health.

The VAS is a vertical scale, similar to one on a thermometer, anchored between the best and worst imaginable health states. In a VAS survey, 100 represents full health as the best imaginable health state, and 0 represents death as the worst imaginable health state. Patients were asked to rate their current health states on the scale, and their utility states were calculated by dividing a participant-selected rate by 100 [23]. The EQ-5D-3L scale is a generic preference measure with five dimensions: mobility, self-care, usual activity, pain/discomfort, and anxiety and depression. Each dimension is rated according to three levels of severity: no problem, some problems, and extreme problems. Having the patients complete the EQ-5D-3L instrument enabled us to obtain five-digit codes that we then converted into utility values on the basis of a health value set [25]. To determine the patients' utility values, we used a validated Persian version of the EQ-5D-3L scale and an Iranian EQ-5D-3L value set, recently published [26].

\subsubsection{Willingness-to-Pay (WTP) Measurements WTP} refers to the maximum amount of money an individual would be willing to pay to obtain a benefit or avoid a detriment. To determine patients' WTP, we asked them to consider a hypothetical situation wherein a new safe treatment that causes no pain and side effects is available; the treatment can cure their disease and comorbidities immediately and enable them to fully recover health but is not covered by government or health insurance. We then asked respondents whether they would be willing to pay a certain bid amount out of pocket (OOP) [13, 27, 28]. A one-off OOP payment per intervention was chosen to elicit respondents' WTP. We considered OOP payment the most suitable of the possible payment methods (including direct OOP payment, taxation, and insurance premiums) because Iran does not have a well-established taxation system, OOP payment is common in Iran's health system, and, generally, a lack of commitment to pay in subsequent months or years may negate the validity of a stated value. To ascertain their true WTP values, respondents were allowed to borrow or lend money in the scenarios but were reminded of the budget constraints, opportunity costs, and financial effects of their decision on their households [29].

We conducted a pilot test in which the distribution of WTP values was determined using an open-ended questionnaire and 15 patients with diabetes from different settings were interviewed to validate the questionnaire and assess the feasibility of the study. After evaluation, we made some modifications to the WTP section of the final questionnaire, including the selection of an appropriate WTP question format among common approaches and the determination of bid values. There are four major approaches to eliciting WTP in a contingent valuation method (CVM): an open-ended method, a dichotomous choice (DC) approach, a bidding game, and the use of a payment card [30]. The DC approach is a binary question format or "referendum format" recommended by the National Oceanic and Atmospheric Administration panel for contingent valuation [31]. It is also commonly used in healthcare studies because of its efficiency and higher response rate, greater realism, and lower strategic bias compared with other CVMs [32]. The DC approach has different formats according to the number of binary questions asked. In our survey, the patients' WTP values were measured using a mixed model or, more specifically, a double-bounded DC (DBDC) method with a follow-up open-ended question. The DBDC method involves the presentation of initial and second bids, with the latter being greater or lower than the former, depending on respondents' answers: If the answer on the WTP question for the initial bid is "yes," the second bid increases and vice versa [33]. After the second bid was provided to respondents, the follow-up open-ended question was presented to draw a more precise estimate of the WTP amount [28]. We also used ex-ante and ex-post approaches to elicit the true WTP. In the ex-ante approach, we reminded patients of their budget constraints; in the ex-post approach, we asked respondents how they would finance their stated WTP [34]. We offered respondents multiple options to choose from, including current income, savings, investments, sale of properties, lending or borrowing, and reducing household expenditures, etc. Respondents were allowed to change their maximum WTP after this consideration.

To reduce or eliminate starting point bias in the DBDC method, we designed nine different starting points based on actual costs of treatments and the WTP values stated in the pilot test (Table 1). To allocate an equal number of occurrences to each starting bid in the questionnaire and avoid the risk of greater or lower occurrences of certain bids, a series of random numbers between one and nine in 17 blocks were generated in Microsoft Excel using the program's RAND function. According to this procedure, the WTP question in the questionnaire was assigned an exclusive starting bid. 
Table 1 Structure of the designed bid values in the double-bounded dichotomous-choice questions

\begin{tabular}{llrc}
\hline Starting point & $\begin{array}{l}\text { Bid 1 } \\
\text { Initial bid }\end{array}$ & $\begin{array}{l}\text { Yes } \\
\text { Bid up }\end{array}$ & \multicolumn{1}{l}{$\begin{array}{l}\text { No } \\
\text { Bid down }\end{array}$} \\
\hline 1 & 169 & 338 & 85 \\
2 & 338 & 1014 & 169 \\
3 & 1014 & 1690 & 338 \\
4 & 1690 & 2366 & 1014 \\
5 & 2366 & 3043 & 1690 \\
6 & 3043 & 3719 & 2366 \\
7 & 3719 & 5071 & 3043 \\
8 & 5071 & 6761 & 3719 \\
9 & 6761 & 10,142 & 5071 \\
\hline
\end{tabular}

This randomization procedure is partly described in Table 2, and more details regarding the RAND function are available in the ESM.

2.3.1.3 Patient Characteristics All the variables used in the study were based on the patients' reports. Factors such as age, sex, marital status, household position, education level, job status, monthly household expenditure as a proxy for income, and health insurance status were examined as demographic and socioeconomic variables. Factors such as diabetes type (T1DM $=$ onset before 30 years of age and currently treated with insulin, T2DM = adult-onset, treated with non-insulin medicines and/or insulin), treatment type (insulin or non-insulin), diabetes duration, and complications and comorbidities (e.g., cardiovascular diseases, hypertension, high cholesterol, kidney diseases, eye diseases, diabetic foot problems, and stroke) were used as disease-related variables. Other factors included the patients' hospitalization experiences in the past year and the near-death experiences of family members in the last year.

\subsection{Data Analysis}

To estimate patients' WTP amount per QALY, we used a chained approach methodology [5], in which Eq. 1 [13, 35] was implemented in two steps: to elicit the current utility weight of a patient and to determine the WTP for improvement of a current health state to full health.

$\frac{\text { WTP }}{\text { QALY }}=\frac{\text { willingness to pay amount }}{\sum_{t=1}^{\text {life expectancy }}(1-\text { current utility }) *(1+r)^{-(t-1)}}$, where $r$ is the discount rate and $t$ represents the remaining lifetime, which varied according to respondent's age group. The equation was used to calculate the ratio of WTP to QALY gained for each respondent, and the mean WTP for QALY was computed using the mean of the ratios. Given that the estimated values were likely to be sensitive to the choice of discount rates, we explored the effects of different rates $(0-7 \%)$ in the QALY value estimation.

Ordinary least squares (OLS) was used to probe into the effects of patient characteristics (e.g., socioeconomic, demographic, and disease-related variables) on WTP for QALYs. Because the assumption of normality had been violated in other WTP studies, we performed a natural log transformation of the dependent variable (WTP/QALY) in the regression analysis to generate a normal distribution and reduce violations of the parametric assumptions underlying the regression analysis. A percentage of $\leq 5 \%$ was considered statistically significant. Microsoft Excel 2010, IBM SPSS version 22, and Stata/MP version 13 were used in the statistical analysis.

\section{Results}

\subsection{Patient Characteristics}

Table 3 presents the general and disease-related characteristics of the patients. In total, $55 \%$ were female, $45 \%$ were male, and the mean age was 56 years. Half of the patients had a high school diploma or higher education; $83 \%$ had T2DM; and $67 \%$ had been admitted to the hospital's outpatient department. Comparison of the disease-related variables showed that patients with T1DM were younger (aged 40 years), had a higher probability of being inpatients, and had had diabetes for a longer duration. All patients with T1DM and 59\% of those with T2DM used insulin. With the exception of hospitalization experiences in the past year, a higher rate of comorbidities and complications was observed among patients with T2DM than those with T1DM. In both patients with T1DM and T2DM, the most common comorbidities were diabetic foot diseases, followed by high cholesterol and eye diseases. These results align with World Health Organization (WHO) fact sheets on the leading causes of diabetes complications, including blindness and amputations [36].

Table 2 Example random allocation of bids to the willingness-to-pay question in the questionnaire

\begin{tabular}{llllllllll}
\hline Questionnaire no. & 1 & 2 & 3 & 4 & 5 & 6 & 7 & 8 & 9 \\
\hline Starting bid & 1690 & 3719 & 338 & 1014 & 5071 & 6761 & 2366 & 3043 & 169 \\
\hline
\end{tabular}


Table 3 General and disease-related characteristics of patients with diabetes

\begin{tabular}{|c|c|c|}
\hline \multicolumn{3}{|l|}{ General characteristics $(n=149)$} \\
\hline Age range, years & $18-85$ & \\
\hline Mean \pm SD age & $56 \pm 13.4$ & \\
\hline Female & $55 \%$ & \\
\hline \multicolumn{3}{|l|}{ Education } \\
\hline Illiterate & $11 \%$ & \\
\hline Under diploma & $38 \%$ & \\
\hline Diploma and university education & $51 \%$ & \\
\hline \multicolumn{3}{|l|}{ Household monthly income (\$US) } \\
\hline$<169$ & $12 \%$ & \\
\hline $169-676$ & $55 \%$ & \\
\hline$>676$ & $33 \%$ & \\
\hline $\begin{array}{l}\text { Near-death experiences of family members in } \\
\text { the past year }\end{array}$ & $16 \%$ & \\
\hline Disease-related characteristics & T1DM & $\mathrm{T} 2 \mathrm{DM}$ \\
\hline Diabetes type & $17 \%$ & $83 \%$ \\
\hline Diabetes duration, year & 18 & 10 \\
\hline Insulin consumption & $100 \%$ & $59 \%$ \\
\hline Inpatient admission & $53 \%$ & $33 \%$ \\
\hline History of cerebral vascular accident & $4 \%$ & $7 \%$ \\
\hline Diabetic foot problems & $54 \%$ & $62 \%$ \\
\hline Kidney disease & $32 \%$ & $38 \%$ \\
\hline Eye disease & $48 \%$ & $50 \%$ \\
\hline Cardiovascular disease & $32 \%$ & $38 \%$ \\
\hline Hypertension & $28 \%$ & $50 \%$ \\
\hline High cholesterol & $48 \%$ & $59 \%$ \\
\hline Hospitalization experiences in the past year & $60 \%$ & $42 \%$ \\
\hline
\end{tabular}

$S D$ standard deviation, $T 1 D M$ type 1 diabetes mellitus, T2DM type 2 diabetes mellitus

Table 4 Health-utility weights derived via different health-utility measures

\begin{tabular}{lll}
\hline Health-utility measure & $\begin{array}{l}\text { Current } \\
\text { health-utility } \\
\text { weight }^{\mathrm{a}}\end{array}$ & $\begin{array}{l}\text { \% of respondents who rated/ } \\
\text { stated current health as full } \\
\text { health state }\end{array}$ \\
\hline EQ-5D-3L & $0.51 \pm 0.24$ & 6 \\
VAS & $0.61 \pm 0.19$ & 2 \\
TTO-adjusted & $0.70 \pm 0.26$ & 22 \\
TTO-10 years & $0.73 \pm 0.28$ & 38 \\
\hline
\end{tabular}

EQ-5D-3L EuroQoL-5 Dimensions 3 Levels, TTO time trade-off, VAS visual analogue scale

${ }^{a}$ Data in this column are presented as mean \pm standard deviation

\subsection{Patient Health-Utility Weights}

Table 4 presents the health-utility weights derived through different utility measures. The mean health-utility weight of patients was $>0.50$, no matter what instrument we used, which may be attributed to the patient characteristics included in the analysis. The EQ-5D-3L and 10-year TTO measurements yielded the lowest and highest health utility values. The results of the TTO technique possibly originated from the patients who were non-traders, that is, those who refused to trade any duration of lifespan in exchange for restoration to full health. To further illustrate this, in the adjusted TTO, 22\% of the respondents refused to trade any time for recovering to full health, and the percentage of nontraders rose to $38 \%$ as the lifetime was reduced to 10 years. Because of the equality in duration characterized by full health and current health states, the utility weight of the nontraders as a proportion of the two durations was one. As a result, their valuations affected the mean value of their utility weights and increased the mean overall. This unwillingness to trade was driven by three important reasons: the interests of the patients' children and families, the non-severity of their health states, and the treatment they are currently undergoing for increased longevity.

\subsection{Patient WTP}

The mean value of WTP for the hypothetical treatment was estimated at $\$$ US 11,220. Among the respondents, 96\% exhibited a positive WTP, and $4 \%$ had a zero WTP because of an inability to pay. Surprisingly, all except one non-trader had a positive WTP for benefits from a permanent cure. The $66 \%$ of the respondents who exhibited a positive WTP responded "yes" to the first bid; however, the proportion of "yes" responses declined from 82 to $56 \%$ as the bid value rose from the lowest (\$US169) to the highest (\$US6761).

\subsection{WTP per Quality-Adjusted Life-Year}

The monetary value of a QALY was estimated using Eq. 1, in which each respondent's WTP for restoration to full health and the amount of QALY gained (the difference between the utility weights of current health and full health) were combined. In all the estimations, the respondents with a health utility value of one (zero QALYs gained) were excluded from the calculations to avoid ending with an undefined value (given a zero value in the denominator in Eq. 1). The value of a QALY ranged from \$US1191 to 2484 when no discount rate was used (Table 5). Using no discount rate could mean that a QALY gained today has the equal value of a QALY in future years, whereas, in reality, individuals prefer to assign different values to benefits during different periods of time, and the future value is less than present because of uncertainty surrounding experiencing future benefits, inflation rates, and time value of money. Therefore, in the context of economic evaluations, most pharmacoeconomic guidelines and government and regulatory agencies recommend using positive discount rates for discounting costs and health benefits. In spite of general consensus on 
Table 5 Mean willingness to pay for quality-adjusted life-years among patients with diabetes and the ratio of mean willingness to pay to gross domestic product per capita

\begin{tabular}{lllll}
\hline Health utility measure & EQ-5D-3L & TTO-adjusted & TTO-10 years & VAS \\
\hline WTP/QALY (\$US) ( $r$, ratio to GDP per capita) & $1191(0.00,0.23)$ & $2484(0.00,0.47)$ & $1899(0.00,0.36)$ & $2088(0.00,0.39)$ \\
& $1643(0.03,0.31)$ & $3457(0.03,0.65)$ & $2531(0.03,0.48)$ & $2902(0.03,0.55)$ \\
& $1994(0.05,0.38)$ & $4215(0.05,0.80)$ & $3013(0.05,057)$ & $3692(0.05,0.70)$ \\
& $2378(0.07,0.45)$ & $5043(0.07,0.95)$ & $3536(0.07,0.67)$ & $4333(0.07,0.82)$ \\
\hline
\end{tabular}

$r=$ discount rate

Exchange rate: IRR29,580 $=\$$ US1; 2015 local GDP per capita $=\$$ US5219

EQ-5D-3L EuroQoL-5 Dimensions 3 Levels, GDP gross domestic product, IRR Iranian rial, $Q A L Y$ quality-adjusted life-year, TTO time trade-off, $V A S$ visual analogue scale, $W T P$ willingness to pay

using the time preference, the choice of discount rate is a critical consideration that is decision-context dependent. The recommended positive discount rates vary in national economic evaluation guidelines, but $5 \%$, followed by $3 \%$ are the most common [37]. In this case, performing sensitivity analysis at different discount rates is the most appropriate approach to responding to these variations and examining their effects. Therefore, based on recommendations from the Washington panel on cost effectiveness in health and medicine on discount rates and studies featuring $0-7 \%$ sensitivity, the current study examined sensitivity levels of up to $7 \%$ (Table 5) [38-40]. Interestingly, all the discount analyses indicated a QALY value $<1$ local GDP per capita. According to a World Bank statement, Iran's local GDP per capita in 2015 was \$US5291.

The determinants of the patients' WTP for QALYs were investigated using a robust OLS regression. The results of the analysis of QALY values derived via the TTO-adjusted scenario are provided in Table 6. Our estimation showed monthly household income and hospitalization experiences in the past year were significantly positively associated with WTP/QALY ratios. Thus, wealthier patients and those with hospitalization experiences in the previous year had higher WTP/QALY ratios, even though the model $R^{2}$ value of 0.16 was low. Near-death experiences of family members in the previous year were significantly negatively associated with WTP/QALY ratios.

\section{Discussion}

To the best of our knowledge, this exploratory study is the first to estimate the value of QALYs by considering the perspectives of people with diabetes mellitus. Patients' WTP per QALY ranged from \$US1191 to 5043, which is equal to 0.23-0.95 times the Iranian GDP per capita in 2015 . Although the QALY values changed in accordance with health preferences and discount rates, all the values were lower than one times the GDP per capita. Thus, in contrast to the WHO's recommendation to use one to three times the GDP per capita as a cost-effectiveness threshold, our findings indicated a value close to the lower bound of the GDP per capita and did not support the higher bound of three times GDP per capita. The results are also consistent with
Table 6 Relationship between $\ln$ (WTP/QALY) derived from time trade-off-adjusted technique and patient characteristics

\begin{tabular}{lclrll}
\hline Variable & Coefficient & Robust SE & $t$ & $p>|t|$ & 95\% CI \\
\hline Self-hospitalization experience & 8.87 & 4.35 & 2.04 & $0.04^{*}$ & $0.24-17.49$ \\
Death of family experience & -16.65 & 6.13 & -2.72 & $0.00^{*}$ & -28.80 to -4.50 \\
Monthly household income (\$) & & & & & \\
2 & 16.73 & 6.71 & 2.49 & $0.01^{*}$ & $3.43-30.04$ \\
3 & 21.71 & 7.33 & 2.96 & $0.00^{*}$ & $7.18-36.25$ \\
Constant & 21.46 & 6.59 & 3.26 & $0.00^{*}$ & $8.41-34.50$ \\
\hline
\end{tabular}

$C I$ confidence interval, $S E$ standard error

Number of observations used in the model: 112

$F(4,107): 0.27$

Prob $>F: 0.0001$

$R^{2}: 0.1549$

Root MSE: 23.364

*Significance level: $5 \%$ 
findings from our recent study [41] wherein we estimated WTP for QALYs from the perspectives of patients with cardiovascular disease. All in all, the findings indicated that the WHO's recommendation (i.e., $<3$ times the GDP per capita) should be used with considerable caution and care in decision making and resource allocation for cost-effective interventions. Moreover, our empirical results revealed an important methodological issue in relation to estimating WTP for QALYs through a chained approach (Eq. 1) for patients who rated/stated their current states as corresponding to full health in their health state valuations while having a positive WTP for obtaining treatment. A zero value in the denominator of Eq. 1, which would result in an undefined WTP/ QALY, and exclusion of these patients or inclusion them with an arbitrary value for QALY gained in the denominator, may produce bias in the monetary estimation of QALYs. Byrne et al. [42] asserted that eliminating respondents with very low QALYs can lead to a downward estimation of QALY values, whereas Zhao et al. [9] found that excluding extreme values from the EQ-5D scale did not produce a significant bias in estimated WTP for a QALY. Therefore, we believe more investigation is required to identify and manage bias when direct or indirect health measures are used to elicit QALY gains. We also acknowledge that bias issues are a potential problem with the TTO technique. In our study, few respondents expressed full health valuations in the EQ5D-3L scale and the VAS, but $22-38 \%$ of patients had zero QALYs gained in the adjusted and conventional scenarios of the TTO method. These respondents constitute a significant sample proportion that cannot be ignored. The interests of the patients' families and children, the non-severity of their disease, and ongoing treatment for increased longevity were the three main drivers of a non-trading orientation among some of the respondents. All except one non-trader had a positive WTP for a permanent cure and restoration to full health; that is, they were willing to pay for zero gain, suggesting that, although patients were aware of their health states, they were unwilling to trade any duration of lifespan in exchange for restoration to full health because of the three reasons mentioned above. Attema et al. [43] pointed out that excluding people with an infinite value for life from analyses is inappropriate, and Iezzi et al. [44] recommended that non-traders be retained in analyses by permitting them to trade in small units of time ( $<1$ year). We believe this idea may be useful in evaluating patients' health-related quality of life but is inappropriate when used to estimate WTP for QALYs using a chained method because very small units of QALYs gained in the denominator of Eq. 1 pose the risk of overestimation. Let us take the TTO-adjusted scenario as an example. Under the absence of non-traders, the monetary value of a QALY is \$US4215, and assuming a 0.999 utility value instead of 1 causes the WTP for QALYs to be overestimated at $\$$ US109,872 or 21 GDP per capita. Such a value exceeds the resources of the healthcare system in any country with limited resources and is therefore unacceptable. On the other hand, eliminating respondents with very small QALYs gained could result in a lower estimation of WTP per QALYs [42]. The apparent unsuitability of trading in small units of time and the continued existence of non-trader issues constitutes an analytical challenge. An alternative would be to employ other health state valuation techniques that have fewer problems of full health valuation.

Despite our results, nothing should be taken at face value, as by no means do we claim that our findings represent the absolute value of a QALY for patients with diabetes. More research is needed to further investigate the effects of including or excluding non-traders or respondents with a full health valuation and positive WTP for the monetary value of QALYs. Some of the main limitations of this study are the sample size and sampling technique. Our sample is not representative of people with diabetes in our society because we interviewed only patients who sought treatment from a publicly funded hospital. Patients from other geographical regions and those admitted into private hospitals or clinics were excluded from the research, as were patients with serious health problems, such as foot amputation, blindness, and severe kidney failure, given the nature of TTO questions. The study is also limited in terms of the remaining lifetime that we used to implement the TTO scenarios and calculate QALYs gained. It was not a life expectancy specific to patients with diabetes and was instead based on national tables for different age groups; such patients may have a shortened lifespan. In this case, the QALYs gained may be underestimated if respondents assume a gain in longevity as well as quality of life after they purchase a "cure." Generalizing the findings, especially when it comes to decision making, requires additional surveys with a broader and more representative sample. For informed decision making and efficient resource allocation, more empirical research should be conducted to elicit disease-specific QALY values from the patient's perspective, with particular emphasis on the nature of a disease, that is, whether it is life threatening, chronic, or disabling [9].

\section{Conclusion}

In this study, the mean WTP for QALYs from the perspectives of patients with diabetes varied according to health measures and discount rates. In all the sensitivity analyses, the mean WTP was less than the local GDP per capita. Our findings suggested that the local GDP per capita can be used as a threshold value in the economic evaluation of different programs but that employing the upper bound recommended by the WHO (i.e., $<3$ times the GDP per capita) needs further investigation. Considering the limitations of 
this study, more empirical research involving diverse patient groups from different settings is recommended to precisely elicit the worth of QALYs and juxtapose this with the WHO recommendation as a general guideline at an international level, especially for middle-income countries with limited healthcare resources, such as Iran.

Acknowledgments The authors thank the Research Institute for Endocrine Sciences of Shahid Beheshti University of Medical Sciences and staff of the diabetes clinic at Shahid Taleghani Hospital for their close cooperation.

Author Contributions All authors contributed to the design of aspects of the study, data collection, data analysis, and interpretation of results. $\mathrm{NM}, \mathrm{LZ}$ and SN made major contributions to drafting the manuscript, and $\mathrm{NM}$ and $\mathrm{SN}$ revised the manuscript critically. The final version of the manuscript was seen and approved by all authors.

\section{Compliance with Ethical Standards}

Data Availability Statement The datasets used and/or analysed during the current study are available from the corresponding author on reasonable request.

Funding This study was funded by the HTA office of the Iran Ministry of Health and Medical Education.

Conflict of interest Najme Moradi, Arash Rashidian, Shirin Nosratnejad, Alireza Olyaeemanesh, Leila Zarei, and Marzieh Zanganeh have no conflicts of interest that are directly relevant to the content of this article.

Approval The study protocol was approved by the ethics committee of Shahid Beheshti University of Medical Sciences and was in accordance with the ethical standards of the Declaration of Helsinki.

Informed Consent All patients provided written informed consent before the interviews were conducted.

Open Access This article is distributed under the terms of the Creative Commons Attribution-NonCommercial 4.0 International License (http://creativecommons.org/licenses/by-nc/4.0/), which permits any noncommercial use, distribution, and reproduction in any medium, provided you give appropriate credit to the original author(s) and the source, provide a link to the Creative Commons license, and indicate if changes were made.

\section{References}

1. Taylor RS, Drummond MF, Salkeld G, Sullivan SD. Inclusion of cost effectiveness in licensing requirements of new drugs: the fourth hurdle. BMJ (Clinical research ed). 2004;329(7472):972-5.

2. Edejer TT-T, Baltussen R, Adam T, Hutubessy R, Acharya A, Evans D, et al. WHO guide to cost-effectiveness analysis. 2012.

3. Eichler H-G, Kong SX, Gerth WC, Mavros P, Jönsson B. Use of cost-effectiveness analysis in health-care resource allocation decision-making: how are cost-effectiveness thresholds expected to emerge? Value Health. 2004;7(5):518-28.
4. Ryen L, Svensson M. The willingness to pay for a quality adjusted life year: a review of the empirical literature. Health Econ. 2015;24(10):1289-301.

5. Robinson A, Gyrd-Hansen D, Bacon P, Baker R, Pennington M, Donaldson C, et al. Estimating a WTP-based value of a QALY: the 'chained' approach. Soc Sci Med. 1982;2013(92):92-104.

6. Nimdet K, Chaiyakunapruk N, Vichansavakul K, Ngorsuraches S. A systematic review of studies eliciting willingness-to-pay per quality-adjusted life year: does it justify CE threshold? PLoS One. 2015;10(4):e0122760.

7. Bobinac A, van Exel J, Rutten FFH, Brouwer WBF. The value of a QALY: individual willingness to pay for health gains under risk. PharmacoEconomics. 2014;32(1):75-86.

8. Lim YW, Shafie AA, Chua GN. Determination of cost-effectiveness threshold For Malaysia. Value Health. 2014;17:438.

9. Zhao F-L, Yue M, Yang H, Wang T, Wu J-H, Li S-C. Willingness to pay per quality-adjusted life year: is one threshold enough for decision-making?: results from a study in patients with chronic prostatitis. Med Care. 2011;49(3):267-72.

10. Brazier J, Akehurst R, Brennan A, Dolan P, Claxton K, McCabe $\mathrm{C}$, et al. Should patients have a greater role in valuing health states? Appl Health Econ Health Policy. 2005;4(4):201-8.

11. Danzon PM, Nicholson S, Towse A, Drummond M, Sorenson C. Measuring value: pharmacoeconomics theory and practice. New York: Oxford University Press; 2012.

12. Pyne JM, Fortney JC, Tripathi S, Feeny D, Ubel P, Brazier J. How bad is depression? Preference score estimates from depressed patients and the general population. Health Serv Res. 2009;44(4):1406-23.

13. King JT, Tsevat J, Lave JR, Roberts MS. Willingness to pay for a quality-adjusted life year: implications for societal health care resource allocation. Med Decis Making. 2005;25(6):667-77.

14. Blumenschein K, Johannesson M. Relationship between quality of life instruments, health state utilities, and willingness to pay in patients with asthma. Ann Allergy Asthma Immunol. 1998;80(2):189-94.

15. Cunningham SJ, Hunt NP. Relationship between utility values and willingness to pay in patients undergoing orthognathic treatment. Commun Dent Health. 2000;17(2):92-6.

16. Zethraeus N. Willingness to pay for hormone replacement therapy. Health Econ. 1998;7(1):31-8.

17. Lundberg L, Johannesson M, Silverdahl M, Hermansson C, Lindberg M. Quality of life, health-state utilities and willingness to pay in patients with psoriasis and atopic eczema. Br J Dermatol. 1999;141(6):1067-75.

18. Lieu TA, Ray GT, Ortega-Sanchez IR, Kleinman K, Rusinak $\mathrm{D}$, Prosser LA. Willingness to pay for a QALY based on community member and patient preferences for temporary health states associated with herpes zoster. PharmacoEconomics. 2009;27(12):1005-16.

19. Narayan KM, Gregg EW, Fagot-Campagna A. Diabetes-a common, growing, serious, costly, and potentially preventable public health problem. Diabetes Res Clin Pract. 2000;2:77-84 (50 Suppl.).

20. Zhang P, Engelgau MM, Norris SL, Gregg EW, Narayan KMV. Application of economic analysis to diabetes and diabetes care. Ann Intern Med. 2004;140(11):972-7.

21. Tolley K. What are health utilities. London: Hayward Medical Communications; 2009.

22. Edejer TT. T. Making choices in health: WHO guide to costeffectiveness analysis. Geneva: World Health Organization; 2003.

23. Brazier J, Deverill M, Green C, Harper R, Booth A. A review of the use of health status measures in economic evaluation. Health Technol Assess. 1999;3(9):i-iv. 
24. Khosravi A, Taylor R, Naghavi M, Lopez AD. Mortality in the Islamic Republic of Iran, 1964-2004. Bull World Health Organ. 2007;85(8):607-14.

25. Oppe M, Devlin NJ, Szende A. EQ-5D value sets: inventory, comparative review and user guide. Dordrecht, Netherland: Springer; 2007.

26. Goudarzi R, Zeraati H, Akbari Sari A, Rashidian A, Mohammad K. Population-Based Preference Weights for the EQ-5D Health States Using the Visual Analogue Scale (VAS) in Iran. Iran Red Crescent Med J. 2016;18(2):e21584.

27. Donaldson C, Baker R, Mason H, Pennington M, Bell S, Lancsar E, et al. European value of a quality adjusted life year-final publishable report 2010. http://research.ncl.ac.uk/eurovaq/EuroV aQ_Final_Publishable_Report_and_Appendices.pdf. Accessed 20 Dec 2018.

28. Smith RD. The relative sensitivity of willingness-to-pay and timetrade-off to changes in health status: an empirical investigation. Health Econ. 2001;10(6):487-97.

29. Smith RD. Construction of the contingent valuation market in health care:a critical assessment. Health Econ. 2003;12(8):609-28.

30. Venkatachalam $L$. The contingent valuation method: a review. Environ Impact Assess Rev. 2004;24(1):89-124.

31. Arrow K, Solow R, Washington DC. Report of the NOAA panel on contingent valuation. National Oceanic and Atmospheric Administration. 1993.

32. Smith RD. The discrete-choice willingness-to-pay question format in health economics: should we adopt environmental guidelines? Med Decis Making. 2000;20(2):194-206.

33. Venkatachalam S, Bagratee JS, Moodley J. Medical management of uterine fibroids with medroxyprogesterone acetate (Depo Provera): a pilot study. J Obstet Gynaecol. 2004;24(7):798-800.

34. Whitehead JC, Cherry TL. Willingness to pay for a Green Energy program: A comparison of ex-ante and ex-post hypothetical bias mitigation approaches. Resour Energy Econ. 2007;29:247-61.
35. Thavorncharoensap M, Teerawattananon Y, Natanant S, Kulpeng $\mathrm{W}$, Yothasamut J, Werayingyong P. Estimating the willingness to pay for a quality-adjusted life year in Thailand: does the context of health gain matter? ClinicoEcon Outcomes Res CEOR. 2013;5:29-36.

36. World Health Organization. 10 Facts about diabetes. https://www. who.int/features/factfiles/diabetes/01_en.html. Accessed 20 Oct 2018.

37. Attema AE, Brouwer WBF, Claxton K. Discounting in economic evaluations. PharmacoEconomics. 2018;36(7):745-58.

38. Mahboub-Ahari A, Pourreza A, Sari AA, Rahimi Foroushani A, Heydari H. Stated time preferences for health: a systematic review and meta analysis of private and social discount rates. $\mathrm{J}$ Res Health Sci. 2014;14(3):181-6.

39. Weinstein MC, Siegel JE, Gold MR, Kamlet MS, Russell LB. Recommendations of the panel on cost-effectiveness in health and medicine. JAMA. 1996;276(15):1253-8.

40. Shiroiwa T, Sung YK, Fukuda T, Lang HC, Bae SC, Tsutani K. International survey on willingness-to-pay (WTP) for one additional QALY gained: what is the threshold of cost effectiveness? Health Econ. 2010;19(4):422-37.

41. Moradi N, Rashidian A, Rasekh HR, Olyaeemanesh A, Foroughi M, Mohammadi T. Monetary value of quality-adjusted life years (QALY) among patients with cardiovascular disease: a willingness to pay study (WTP). Iran J Pharm Res IJPR. 2017;16(2):823-33.

42. Byrne MM, O'Malley K, Suarez-Almazor ME. Willingness to pay per quality-adjusted life year in a study of knee osteoarthritis. Med Decis Making. 2005;25(6):655-66.

43. Attema AE, Edelaar-Peeters Y, Versteegh MM, Stolk EA. Time trade-off: one methodology, different methods. Eur J Health Econ. 2013;14(Suppl 1):S53-64.

44. Iezzi A, Richardson J. Measuring quality of life at the center for health economics, description of instruments, interview props and their administration. Centre for Health Economics, Monash University, Melbourne; 2009.

\section{Affiliations}

\section{Najme Moradi ${ }^{1}$ - Arash Rashidian ${ }^{2} \cdot$ Shirin Nosratnejad $^{3} \cdot$ Alireza Olyaeemanesh $^{4,5} \cdot$ Marzieh Zanganeh $^{6}$. Leila Zarei ${ }^{7}$}

1 Health Management and Economics Research Center, Iran University of Medical Sciences, Tehran, Iran

2 Department of Health Management and Economics, School of Public Health, Tehran University of Medical Sciences, Tehran, Iran

3 Department of Health Economics, Iranian Centre of Excellence in Health Service Management, Tabriz University of Medical Sciences, Tabriz, Iran
4 Health Economics Department, National Institute for Health Research, Tehran University of Medical Sciences, Tehran, Iran

5 Health Equity Research Centre, Tehran University of Medical Sciences, Tehran, Iran

6 Head of Health Insurance Office, Ministry of Health and Medical Education, Tehran, Iran

7 Health Policy Research Center, Institute of Heath, Shiraz University of Medical Sciences, Tehran, Iran 